OPEN ACCESS

Edited by:

Wei Gu,

University of

Luxembourg, Luxembourg

Reviewed by:

Lina Ma,

Capital Medical University, China Junfang Chen,

Fudan University, China

*Correspondence:

Olivier Beauche

olivier.beauchet@umontreal.ca

Specialty section:

This article was submitted to

Dementia and Neurodegenerative

Diseases,

a section of the journa

Frontiers in Neurology

Received: 30 August 2021 Accepted: 13 December 2021

Published: 06 January 2022

Citation:

Beauchet O, Cooper-Brown LA,

Lubov J, Allali G, Afilalo M and Launay CP (2022) "Emergency Room Evaluation and Recommendations" (ER2) Tool for the Screening of Older Emergency Department Visitors With Major Neurocognitive Disorders: Results From the ER ${ }^{2}$ Database

Front. Neurol. 12:767285.

doi: 10.3389/fneur.2021.767285

\section{"Emergency Room Evaluation and Recommendations" (ER2) Tool for the Screening of Older Emergency Department Visitors With Major Neurocognitive Disorders: Results From the ER ${ }^{2}$ Database}

\author{
Olivier Beauchet ${ }^{1,2,3,4 *}$, Liam A. Cooper-Brown ${ }^{5}$, Joshua Lubov ${ }^{3}$, Gilles Allali ${ }^{6,7}$, \\ Marc Afilalo ${ }^{8}$ and Cyrille P. Launay ${ }^{3}$
}

${ }^{1}$ Department of Medicine, University of Montreal, Montreal, QC, Canada, ${ }^{2}$ Research Center of the Geriatric University Institute of Montreal, Montreal, QC, Canada, ${ }^{3}$ Division of Geriatric Medicine, Department of Medicine, Sir Mortimer B. Davis Jewish General Hospital, Lady Davis Institute for Medical Research, McGill University, Montreal, QC, Canada, ${ }^{4}$ Lee Kong Chian School of Medicine, Nanyang Technological University, Singapore, Singapore, ${ }^{5}$ Faculty of Medicine, McGill University, Montreal, QC, Canada, ${ }^{6}$ Division of Neurology, Department of Neurosciences, Geneva University Hospitals, Geneva, Switzerland, ${ }^{7}$ Leenaards Memory Center, Lausanne University Hospital, University of Lausanne, Lausanne, Switzerland, ${ }^{8}$ Emergency Department, Jewish General Hospital, McGill University, Montreal, QC, Canada

Purpose: The Emergency Room Evaluation and Recommendation $\left(E R^{2}\right)$ is an application in the electronic medical file of patients visiting the Emergency Department (ED) of the Jewish General Hospital (JGH; Montreal, Quebec, Canada). It screens for older ED visitors at high risk of undesirable events. The aim of this study is to examine the performance criteria (i.e., sensitivity, specificity, positive predictive value [PPV], negative predictive value [NPV], positive likelihood ratio [LR+], negative likelihood ratio [LR-] and area under the receiver operating characteristic curve [AUROC]) of the $\mathrm{ER}^{2}$ high-risk level and its "temporal disorientation" item alone to screen for major neurocognitive disorders in older ED visitors at the JGH.

Methods: Based on a cross-sectional design, 999 older adults (age $84.9 \pm 5.6,65.1 \%$ female) visiting the ED of the $\mathrm{JGH}$ were selected from the $\mathrm{ER}^{2}$ database. $\mathrm{ER}^{2}$ was completed upon the patients' arrival at the ED. The outcomes were $\mathrm{ER}^{2}$ 's high-risk level, the answer to $\mathrm{ER}^{2}$ 's temporal disorientation item (present vs. absent), and the diagnosis of major neurocognitive disorders (yes vs. no) which was confirmed when it was present in a letter or other files signed by a physician.

Results: The sensitivities of both $\mathrm{ER}^{2}$ 's high-risk level and temporal disorientation item were high (>0.91). Specificity, the PPV, LR+, and AROC were higher for the temporal disorientation item compared to $\mathrm{ER}^{2}$ 's high-risk level, whereas a highest sensitivity, LR-, and NPV were obtained with the $\mathrm{ER}^{2}$ high-risk level. Both area under the receiver operating characteristic curves were high $\left(0.71\right.$ for $E R^{2}$ 's high-risk level and 0.82 for $E R^{2}$ temporal disorientation item). The odds ratios (OR) of $E R^{2}$ 's high-risk level and of temporal disorientation item for the diagnosis of major neurocognitive disorders were 
positive and significant with all OR above 18, the highest OR being reported for the temporal disorientation item in the unadjusted model $[\mathrm{OR}=26.4$ with $95 \%$ confidence interval $(\mathrm{Cl})=17.7-39.3]$.

Conclusion: Our results suggest that $\mathrm{ER}^{2}$ and especially its temporal disorientation item may be used to screen for major neurocognitive disorders in older ED users.

Keywords: emergency department, major neurocognitive disorders, screening, older adults, performance evaluation

\section{INTRODUCTION}

Major neurocognitive disorders are common in older emergency department (ED) visitors, with a prevalence up to $30 \%(1-4)$. These disorders are associated with a high risk of undesirable events. For instance, older Canadian patients with major neurocognitive disorders are 1.5 times more likely to experience undesirable events, such as delirium, falls and, prolonged length of stay, compared to their cognitively healthy counterparts (5). The presence of major neurocognitive disorders is often unknown upon arrival to the ED (1-3). However, detecting major neurocognitive disorders early is helpful to initiate the appropriate management and prevention strategies $(4,5)$. Therefore, screening for major neurocognitive disorders in older ED patients may limit the occurrence of delirium or other undesirable hospital-related events and is thus recommended early in the hospital care process (4).

Hospital admission is often preceded by an ED visit in older adults (6-8). Therefore, screening for major neurocognitive disorders should systematically be included as a step in assessing older patients upon arrival to the ED. There are obstacles to implementing systematic screenings of major neurocognitive disorders in EDs $(9,10)$. First, EDs are under duress because of overcrowding, delays, and diversions, which have increased to epidemic proportions (6). In Canada, these ED features are exacerbated by the scarce number of hospital beds per capita, which is one of the lowest among Organization for Economic Cooperation and Development (OECD) nations ${ }^{1}$. Second, EDs are configured for the care of single acute diseases, rather than chronic diseases associated with disabilities like Alzheimer's disease, the most common cause of major neurocognitive disorders $(6,7)$. Third, human resources, including the experience and demands on time of ED staff, represent a practical barrier to completing cognitive assessments $(3,4)$. Fourth, the prevalence of undiagnosed Alzheimer's disease amongst older patients may be as high as $90 \%$ (11).

The screening of major neurocognitive disorders needs to be compatible with the daily practice of ED staff. The Emergency Room Evaluation and Recommendations $\left(\mathrm{ER}^{2}\right)$ tool is a validated clinical assessment that screens for older ED users at a high risk of undesirable events $(12,13)$. The tool is composed of 6 items (age, sex, use of home support, polypharmacy, use of walking aid and inability to give month and/or year), providing a score ranging from 0 to 14 and classifying patients into three risk

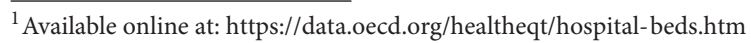

levels: low, moderate, and high. The inability to give month and/or year is the only $\mathrm{ER}^{2}$ item which explores cognition. Temporal disorientation is frequently affected at advanced stages of neurocognitive disorders and, thus, this item could be used to screen individuals with undiagnosed major neurocognitive disorders, regardless of their etiology (3, 4, 9, 11). Since 2017, $\mathrm{ER}^{2}$ has been included in the electronic medical record of ED patients at the Jewish General Hospital (JGH, Montreal, Quebec, Canada) as part of clinical quality improvement program. In this hospital, $\mathrm{ER}^{2}$ is used by $\mathrm{ED}$ nurses like a application to assess older ED users on stretchers upon their arrival. Its completion takes $<3 \mathrm{~min}$, after which the $\mathrm{ER}^{2}$ score and risk level are automatically provided (12). Thus, $\mathrm{ER}^{2}$ is a quick assessment which is compatible with the ED daily practice usually characterized by a huge workflow. In addition, ER ${ }^{2}$ helps ED staff to early consider the most relevant geriatric syndromes in order to make the best choices for the older ED patients $(12,13) . E^{2}$ is, thus, used as a triage tool for older ED users. All information collected from the electronic medical record of older ED users is recorded in a database with the agreement of the Research Review Office of the Jewish General Hospital and the Research Ethics Committee of the Integrated Health and Social Services University Network for West-Central Montreal (Montreal, Quebec, Canada). The $\mathrm{ER}^{2}$ database represents an opportunity to test the hypothesis that the $\mathrm{ER}^{2}$ "high" risk level and/or its "temporal disorientation" item may be used to screen older ED users with major neurocognitive disorders. The aim of this study was to examine the performance criteria (i.e., sensitivity, specificity, positive predictive value [PPV], negative predictive value [NPV], positive likelihood ratios [LR+], negative likelihood ratios [LR-] and area under the receiver operating characteristic curve [AUROC]) of the $\mathrm{ER}^{2}$ high risk level and the temporal disorientation item alone for screening major neurocognitive disorders in older ED users at the JGH.

\section{METHODS}

\section{Study Design and Population}

Based on a cross-sectional design, 999 older adults (age 84.9 $\pm 5.6,65.1 \%$ female) visiting the ED of the JGH (Montreal, Quebec, Canada) were selected from the $\mathrm{ER}^{2}$ database. The inclusion criteria were age $\geq 75$, unplanned $\mathrm{ED}$ visit, being on a stretcher, $\mathrm{ER}^{2}$ information collected and score recorded, and patient agreement for data recording in the $\mathrm{ER}^{2}$ database. The selected participants were patients who consecutively visited the 
JGH ED in the first year (September 2017 to September 2018) of collecting information for the $\mathrm{ER}^{2}$ database.

\section{Emergency Room Evaluation and Recommendations Tool}

$\mathrm{ER}^{2}$ is a component of ED patients' electronic medical record. It is used as an application to automatically calculate the $\mathrm{ER}^{2}$ score and level of risk for undesirable hospital-related events $(12,13)$. Six close-ended questions (i.e., yes vs. no) compose $\mathrm{ER}^{2}$. They are: age 85 and over, male, polypharmacy (defined as number of medications taken daily $\geq 5$ ), use of formal (i.e., health care or social services) and/or informal (i.e., family and/or friend) home support, use of a walking aid regardless of type, and temporal disorientation (defined as inability to name the current month and/or year). A score of five points is assigned to items "use of a walking aid" and "temporal disorientation," while a score of one point is assigned to the other items. The $\mathrm{ER}^{2}$ score ranges from 0 (lowest risk) to 14 (highest risk) and stratifies risk for undesirable events into low (score $0-3$ ), medium (score 4-5) and high (score $\geq 6$ ) risk levels (12).

\section{Assessment of Participant Characteristics}

Participants were assessed by the triage nurse upon their arrival to the ED of the JGH. Information including patients' age, sex, and place of living was thereby collected and recorded in the electronic medical record, as was the Canadian ED Triage and Acuity Scale (14). This scale classifies patients according to the type and urgency of their health condition for prioritization purposes. It is composed of 5 levels of urgency: level 1, defined as resuscitation; level 2, defined as emergent; level 3, defined as urgent; level 4, defined as less urgent; and level 5, defined as non-urgent. Reasons for ED visits were also recorded in the patients' files. In this study, this information was classified into 5 sub-types: Organ failure, defined as an acute organ decompensation; mobility disorders, defined as gait and/or balance impairment and/or fall with or without fallrelated injuries; Neuropsychiatric disorders, defined as delirium, dementia, and/or behavioral disorders; A social issues, defined as the absence of symptoms of acute disease and combined with an acute increase in the use of formal and/or informal home and social services leading to an inability to cope with life circumstances. Once triage was completed and the older $\mathrm{ED}$ user was on a stretcher, a designated nurse completed $\mathrm{ER}^{2}$ at bedside.

For this study, information on major neurocognitive disorders was collected through a review of the patient's digital files by two research assistants. The diagnosis of major neurocognitive disorders was confirmed when it was present in a letter or other files signed by a physician.

\section{Outcomes}

The outcomes were the $\mathrm{ER}^{2}$ "high" risk level, the answer to $\mathrm{ER}^{2}$ 's "temporal disorientation" item (present vs. absent) and the diagnosis of major neurocognitive disorders (yes vs. no).

\section{Participant Consent and Protocol Approval and Registration}

Verbal informed consent was obtained for all participants following a systematic and standardized process used in the ED ward of the JGH. Participants or their legal guardian, when appropriate, were informed that their medical information may be used for research purposes. If they disagreed, they informed the treating physician, and a note was recorded in their chart. The Ethics Committee the Jewish General Hospital approved this process.

\section{Statistical Analysis}

Two research assistants independently conducted data extraction. A consensus procedure was defined in case of disagreement but was not implemented due to concordance. The participant's baseline characteristics were summarized using means, standard deviations (SD), frequencies and percentages, as appropriate. They were separated into two groups: participants with major neurocognitive disorders and those without. Chisquare or unpaired $t$-tests were used to compare groups. The performance criteria (i.e., sensitivity, specificity, PPV, NPV, positive and negative LR and AUROC) of the $\mathrm{ER}^{2}$ high risk level and temporal disorientation item alone for screening major neurocognitive disorders were calculated.

Two separate logistic regression models were constructed to examine the association between major neurocognitive disorders used as dependent variable and the $\mathrm{ER}^{2}$ high risk level and the $\mathrm{ER}^{2}$ temporal disorientation item used as independent variables. $P$-values $<0.05$ were considered statistically significant. All statistics were performed using SPSS (version 23.0; SPSS, Inc., Chicago, IL).

\section{RESULTS}

As shown in Table 1, when comparing older ED users with major neurocognitive disorders to those without, older ED users with major neurocognitive disorders were older, less frequently living at home, and more often had home support, polypharmacy, and temporal disorientation $(P \leq 0.012)$. The reasons for ED visits significantly differed among older ED users with major neurocognitive disorders compared to those without. Those with major neurocognitive disorders less often visited EDs for organ failure and mobility disorders but more frequently for neuropsychiatric disorders and social issues $(P<0.001)$. The distribution of urgency levels was significantly different between groups $(P<0.05)$. Mean $\mathrm{ER}^{2}$ scores were higher in older ED users with major neurocognitive disorders $(P<0.001)$. There were more high-risk patients and fewer at the low risk level among participants with major neurocognitive disorders $(\mathrm{P}<0.001)$. The sensitivities of both $\mathrm{ER}^{2}$ 's high risk level and temporal disorientation were high $(\geq 0.91)$. Specificity, PPV, $\mathrm{LR}+$, and AROC were higher for the temporal disorientation item compared to $\mathrm{ER}^{2}$ 's high risk level, whereas the highest sensitivity, LR-, and NPV were obtained with the $\mathrm{ER}^{2}$ high risk level (Table 2). In addition, both AROC were high ( 0.71 for $\mathrm{ER}^{2}$ 's high-risk level and 0.82 for $\mathrm{ER}^{2}$ temporal disorientation item). 
TABLE 1 | Participant characteristics comparing patients with and without major neurocognitive disorder status ( $n=999)$.

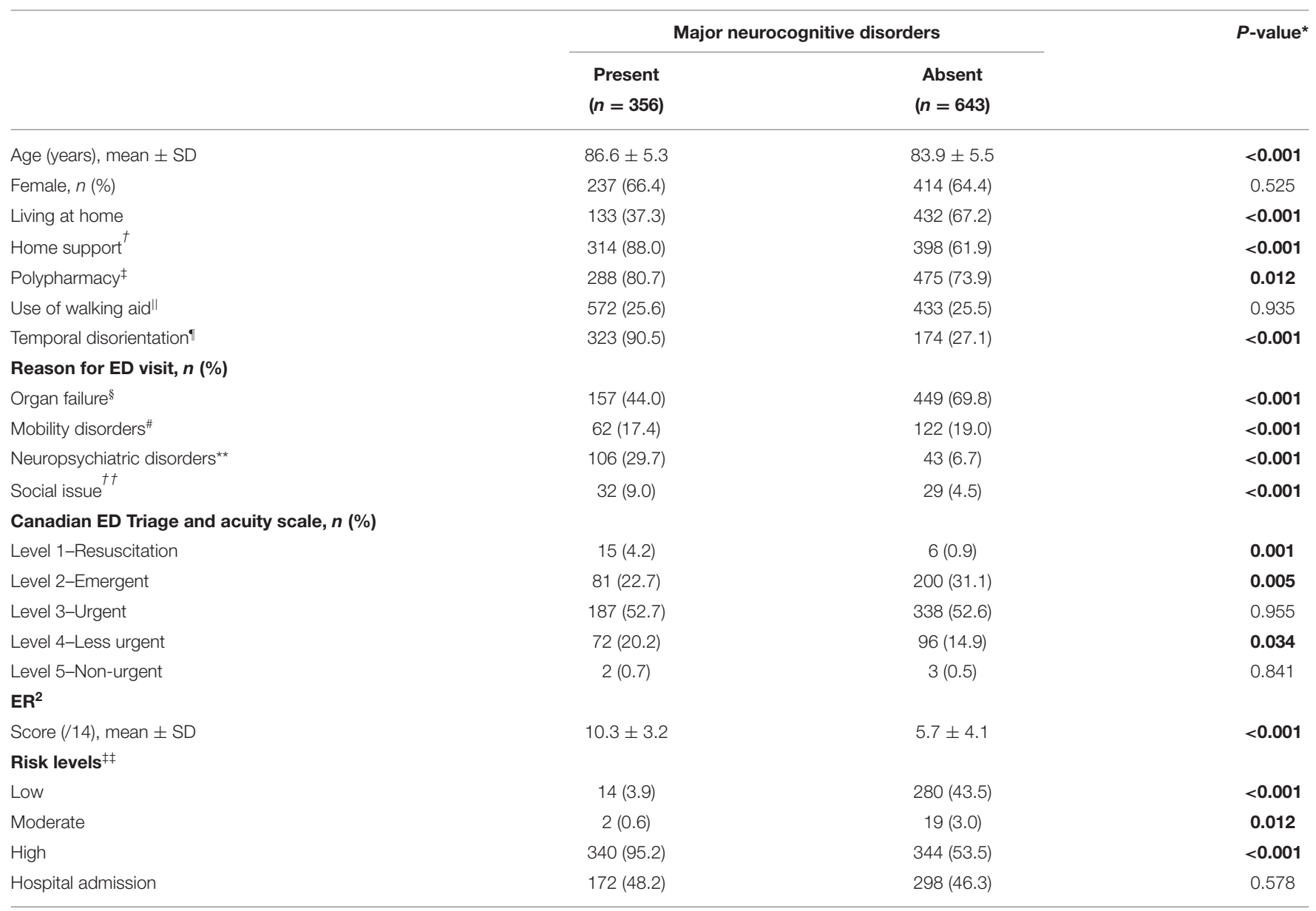

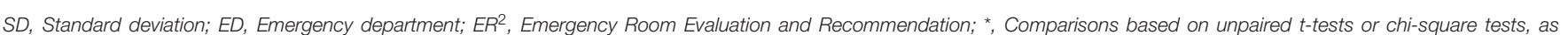

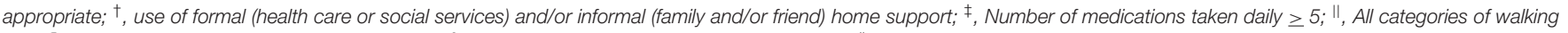

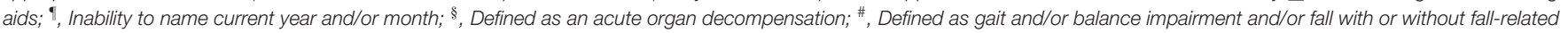

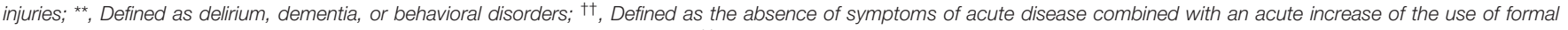

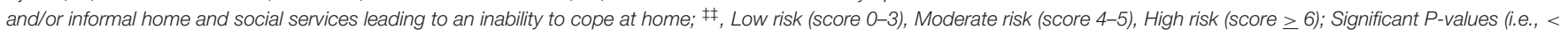
0.05) in bold.

Logistic regressions showed that the odds ratios (OR) of $\mathrm{ER}^{2}$ 's high risk level and of temporal disorientation for the diagnosis of major neurocognitive disorders were positive and significant (all $P$-values $<0.001$ ), with the highest OR being reported for the temporal disorientation item in the unadjusted model [OR = 26.4 with $95 \%$ confidence interval $(\mathrm{CI})=17.7-39.3$ ] (Table 3).

\section{DISCUSSION}

The findings show that $\mathrm{ER}^{2}$ may be used to screen older ED users for their risk of major neurocognitive disorders. Both the $\mathrm{ER}^{2}$ high risk level and the "temporal disorientation" item included in $\mathrm{ER}^{2}$ were able to properly detect these disorders upon arrival to the ED. The "temporal disorientation" item alone showed superior performance to $\mathrm{ER}^{2}$ 's high risk level to predict a major neurocognitive disorder diagnosis, while $\mathrm{ER}^{2}$ 's high risk level was even more sensitive. Additionally, the findings confirm the high prevalence of major neurocognitive disorders of $36.5 \%$ in older ED users. In sum, we found that the inability to give the month and/or year upon arrival to the ED may be useful to screen older ED users for major neurocognitive disorders and easy to use in such busy area.

When it comes to taking care of older ED users, ED staff are concerned with being able to identify the right patients (i.e., those most at risk of adverse outcomes) at the right time (i.e., as soon as possible) and to introduce the right interventions (i.e., those most appropriate to patient health and functional conditions) with the objective of reducing the occurrence of undesirable events (6-8). The best way to prevent or minimize these undesirable events is screening older patients as soon as possible (i.e., earlier in the ED care plan) to establish those at the highest risk and who are candidates for timely and appropriate interventions $(6,10,13)$. Major neurocognitive disorders are underdiagnosed by clinicians and underreported by patients and families, especially in EDs, whereas cognitive impairment/delirium are geriatric conditions 
that must be identified to designed a tailored care plan (1). Only about half of individuals who meet the criteria for major neurocognitive disorders are diagnosed by a clinician $(1,11)$. Furthermore, the diagnosis of major neurocognitive disorders is often delayed, with more older patients diagnosed at advanced stages of neurocognitive disorders, rather than at their onset $(4,9)$. As such, temporal disorientation is frequently observed in this population. This may explain why temporal disorientation can be an efficient way to screen older ED users for major neurocognitive disorders. Firstly, this emphasizes that patients with major neurocognitive disorders at more advanced stages are at greater risk of undesirable events compared to those who are at the onset of these disorders. Thus, it is important to screen the subgroup of patients visiting EDs with more advanced impairments, who are more at risk compared to those at the onset of major neurocognitive disorders (12). Second, an inability to give the month and/or year may be a marker of two types of cognitive impairment: major neurocognitive disorders at moderate to severe stages, as well as delirium (13). Delirium is highly frequent in patients with major neurocognitive disorders, especially in new environments and settings of acute disease, which are two conditions characterizing the $\operatorname{ED}(15,16)$. Third, delirium is a red flag for the underlying presence of major neurocognitive disorders in patients who are otherwise undiagnosed (16). Thus, temporal disorientation should trigger a cognitive assessment, which should consist of two separate

TABLE 2 | Performance criteria of $E R^{2}$ 's high risk level and $E R^{2}$ 's temporal disorientation item for the diagnosis of major neurocognitive disorders $(n=999)$.

\begin{tabular}{lcc}
\hline & \multicolumn{2}{c}{ ER $^{2}$} \\
\cline { 2 - 3 } & $\begin{array}{c}\text { High } \\
\text { risk level }\end{array}$ & $\begin{array}{r}\text { Item temporal } \\
\text { disorientation }\end{array}$ \\
\hline Sensitivity & 0.96 & 0.91 \\
Specificity & 0.47 & 0.73 \\
Positive predictive value & 0.59 & 0.65 \\
Negative predictive value & 0.95 & 0.93 \\
Likelihood ratio of positive test & 1.79 & 3.35 \\
Likelihood ratio of negative test & 10.34 & 7.87 \\
Area under the receiver operating & 0.71 & 0.82 \\
characteristic curve & &
\end{tabular}

$E R^{2}$, Emergency Room Evaluation and Recommendation. processes. First, the diagnosis of delirium must promptly be examined using standardized testing like the Confusion Assessment Method. Second, if the Confusion Assessment Method test is negative, the diagnosis of major neurocognitive disorders may be discussed later in the care process, following ED discharge. Regardless of the clinical diagnosis (i.e., delirium vs. major neurocognitive disorders), it is mandatory to immediately initiate interventions to limit the occurrence of undesirable events $(7,13)$. This last point highlights the advantage of choosing screening tests for cognitive impairment (i.e., delirium vs. major neurocognitive disorders) that are more sensitive than specific, especially as there are few potential adverse effects to interventions aimed to avoid the occurrence of undesirable events. Therefore, we suggest that it would be favorable to use the temporal disorientation item of $\mathrm{ER}^{2}$ as a screening tool for major neurocognitive disorders. Indeed, even if this item may be overly sensitive by classifying patients as having major neurocognitive disorders when they do not, there is a net benefit to introducing interventions that will improve the care of those who are cognitively impaired.

A good screening tool should be based on reliable (i.e., objective, standardized, and communicable) and valid clinical information, which can easily be obtained in busy EDs and used by all ED staff (17). Both $\mathrm{ER}^{2}$ and its "temporal disorientation" item meet these criteria. Recently, we demonstrated the usability of $\mathrm{ER}^{2}$ in EDs and its compatibility with daily ED practice (12). For instance, we reported that the mean time to collect and record $\mathrm{ER}^{2}$ items into a patient's digital chart was $3 \mathrm{~min}$ at bedside. It has been shown that, for a screening tool to be considered usable in the ED setting, personnel must be able to complete it in < $5 \mathrm{~min}$ (18). Moreover, a recent systematic review showed that most tools do not consider usability in their development (19). It is therefore important to note that the $\mathrm{ER}^{2}$ study was classified as a clinical quality improvement program for older ED users. This choice was made to consider the real-life conditions of ED practice, ensuring that $\mathrm{ER}^{2}$ could feasibly be integrated into daily practice by ED nurses.

We observed a high prevalence of major neurocognitive disorders in older ED users, which is consistent with previous studies' findings of up to $30 \%(1-5)$.

There are limitations to consider in our study. Firstly, the study involved a single center, raising the possibility that the studied population may not be representative of all older ED users. This limits the external validity of

TABLE 3 | Regressions showing the association of the diagnosis of major neurocognitive disorders (dependent variable) with ER²'s high risk level and ER²'s temporal disorientation item (independent variables) $(n=999)$.

\begin{tabular}{|c|c|c|c|c|c|c|}
\hline & \multicolumn{3}{|c|}{ Model 1} & \multicolumn{3}{|c|}{ Model 2} \\
\hline & OR & {$[95 \% \mathrm{Cl}]$} & $P$-Value & OR & {$[95 \% \mathrm{Cl}]$} & $P$-Value \\
\hline $\mathrm{ER}^{2}$ high risk level & 18.5 & {$[10.9 ; 31.2]$} & $\leq 0.001$ & 18.4 & [10.0;33.9] & $\leq 0.001$ \\
\hline A ER ${ }^{2}$ temporal disorientation item & 26.4 & {$[17.7 ; 39.3]$} & $\leq 0.001$ & 16.6 & [10.9;25.2] & $\leq 0.001$ \\
\hline
\end{tabular}

$E R^{2}$, Emergency Room Evaluation and Recommendation; ED, Emergency department; OR, Odds ratio; Cl, Confidence interval; Model 1, unadjusted; Model 2, adjusted on age, sex,

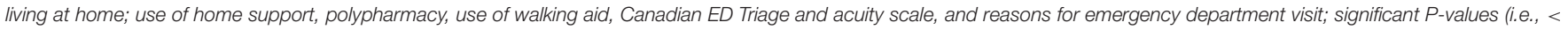
0.05) in bold. 
the results. Secondly, the diagnosis of major neurocognitive disorders was established retrospectively based on information collected in participant's electronic medical records, rather than by an exhaustive examination. As a result, the processes leading to diagnoses of major neurocognitive disorders may be variable and more likely underestimated. Third, the present study examined major neurocognitive disorders as a binary endpoint and, thus, their severity and type could not be considered.

In conclusion, our results suggest that $\mathrm{ER}^{2}$ and, especially, its temporal disorientation item may be used to screen for major neurocognitive disorders in older ED users. There is a need to confirm these results using multicentre observational cohort studies in additional Canadian hospitals.

\section{DATA AVAILABILITY STATEMENT}

Data and materials are available on request. Request should be sent to the corresponding author: Olivier Beauchet, Research Centre of the Geriatric University institute of Montreal, Montreal, QC, Canada; E-mail: olivier.beauchet@umontreal.ca. All requests need a cover letter explaining the objective, justification and the referent ethic committee.

\section{ETHICS STATEMENT}

The Jewish General Hospital (McGill University, Quebec, Canada) Research Ethics Committee approved the study. The Ethics Committee waived the requirement of written informed consent for participation. Verbal informed consent was obtained for all participants.

\section{REFERENCES}

1. Timmons S, Manning E, Barrett A, Brady NM, Browne V, O'Shea E, et al. Dementia in older people admitted to hospital: a regional multi-hospital observational study of prevalence, associations and case recognition. Age Ageing. (2015) 44:993-9. doi: 10.1093/ageing/ afv131

2. Travers C, Byrne GJ, Pachana NA, Klein K, Gray LC. Prospective observational study of dementia in older patients admitted to acute hospitals. Australas J Ageing. (2014) 33:55-8. doi: 10.1111/ajag.12021

3. O'Sullivan D, Brady N, Manning E, O'Shea E, O'Grady S, O 'Regan N, et al. Validation of the 6-Item Cognitive Impairment Test and the 4AT test for combined delirium and dementia screening in older Emergency Department attendees. Age Ageing. (2018) 47:61-8. doi: 10.1093/ageing/ afx149

4. Clevenger CK, Chu TA, Yang Z, Hepburn KW. Clinical care of persons with dementia in the emergency department: a review of the literature and agenda for research. J Am Geriatr Soc. (2012) 60:1742-8. doi: 10.1111/j.1532-5415.2012.04108.x

5. Improving BC's Care For Persons With Dementia In Emergency Departments And Acute Care Hospitals (2011). Available online at: https://www2.gov.bc.ca/assets/gov/people/seniors/about-seniorsbc/ pdf/improvingcaredementiareport2011.pdf

6. Aminzadeh F, Dalziel WB. Older adults in the emergency department: a systematic review of patterns of use, adverse outcomes, and effectiveness of interventions. Ann Emerg Med. (2002) 39:238-47. doi: 10.1067/mem.2002.121523

\section{AUTHOR CONTRIBUTIONS}

OB: principal investigator, study conception and design, obtaining funding, recruitment of participants, analyzing data, drafting first version of the manuscript, and approval of the final manuscript. MA and CL: study design, recruitment of participants, analyzing data, drafting first version, and approval of the final manuscript. LC-B, JL, and GA: revising the manuscript critically for important intellectual content and approval of the final manuscript. All authors contributed to the article and approved the submitted version.

\section{FUNDING}

The study was financially supported by private donations (Oberfeld family) and the Foundation of the Jewish General Hospital (Montreal, QC, Canada). The funding sources have no involvement in study design; in the collection, analysis and interpretation of data; in the writing of the manuscript; and in the decision to submit the article for publication.

\section{ACKNOWLEDGMENTS}

The authors are grateful for their cooperation: (1) the participants; (2) nurses of the Department of emergency of Jewish General hospital, and in particular Mrs Valerie Shneidman and Mr Jonathan Harroche; (3) Mrs. Claude Krinsky from the Foundation of the Jewish General Hospital; (4) the Department of information and technology of Jewish General Hospital, and in particular Mrs Isabelle Aumont, Mrs Maria Veres, Mrs Christine Bougie and Mrs France Guimont; and (5) Oberfeld family.

7. Salvi F, Morichi V, Grilli A, Giorgi R, De Tommaso G, DessìFulgheri P. The elderly in the emergency department: a critical review of problems and solutions. Intern Emerg Med. (2007) 2:292-301. doi: 10.1007/s11739-007-0081-3

8. Šteinmiller J, Routasalo P, Suominen T. Older people in the emergency department: a literature review. Int J Older People Nurs. (2015) 10:284305. doi: 10.1111/opn.12090

9. Calf AH, Pouw MA, van Munster BC, Burgerhof JGM, de Rooij SE, Smidt N. Screening instruments for cognitive impairment in older patients in the emergency department: a systematic review and meta-analysis. Age Ageing. (2021) 50:105-12. doi: 10.1093/ageing/ afaa 183

10. Carpenter CR, Shelton E, Fowler S, Suffoletto B, Platts-Mills TF, Rothman $\mathrm{RE}$, et al. Risk factors and screening instruments to predict adverse outcomes for undifferentiated older emergency department patients: a systematic review and meta-analysis. Acad Emerg Med. (2015) 22:1-21. doi: 10.1111/ac em.12569

11. McGrath R, Robinson-Lane SG, Clark BC, Suhr JA, Giordani BJ, Vincent BM. Self-reported dementia-related diagnosis underestimates the prevalence of older americans living with possible dementia. J Alzheimers Dis. (2021) 82:373-80. doi: 10.3233/JAD-201212

12. Launay CP, Galery K, Vilcocq C, Afilalo M, Beauchet O. Risk for short-term undesirable outcomes in older emergency department users: results of the ER2 observational cohort study. PLoS ONE. (2021) 16:e0249882. doi: 10.1371/journal.pone.0249882

13. Beauchet O, Lubov J, Galery K, Afilalo M, Launay CP. Emergency room evaluation and recommendations for older emergency department users: 
results of the ER ${ }^{2}$ experimental study. Eur Geriatr Med. (2021) 12:9219. doi: 10.1007/s41999-021-00517-0

14. Bullard MJ, Musgrave E, Warren D, Unger B, Skeldon T, Grierson R et al. Revisions to the canadian emergency department triage and acuity scale (CTAS) guidelines 2016. CJEM. (2017) 19:S18-27. doi: 10.1017/cem. 2017.365

15. Oliveira J E Silva L, Berning MJ, Stanich JA, Gerberi DJ, Murad MH, Han JH, et al. Risk factors for delirium in older adults in the emergency department: a systematic review and meta-analysis. Ann Emerg Med. (2021) 8:549-65. doi: 10.1016/j.annemergmed.2021.03.005

16. Zhang XM, Jiao J, Xie XH, Wu XJ. The association between frailty and delirium among hospitalized patients: an updated meta-analysis. J Am Med Dir Assoc. (2021) 22:527-34. doi: 10.1016/j.jamda.202 1.01 .065

17. Marcoux V, Chouinard MC, Diadiou F, Dufour I, Hudon C. Screening tools to identify patients with complex health needs at risk of high use of health care services: a scoping review. PLoS ONE. (2017) 12:e0188663. doi: 10.1371/journal.pone. 0188663

18. Elliott A, Hull L, Conroy SP. Frailty identification in the emergency department-a systematic review focusing on feasibility. Age Ageing. (2017) 46:509-13. doi: 10.1093/ageing/afx019
19. McLean B, Hossain N, Donison V, Gray M, Durbano S, Haase K, et al. Providing medical information to older adults in a web-based environment: systematic review. JMIR Aging. (2021) 4:e24092. doi: 10.2196/24092

Conflict of Interest: The authors declare that the research was conducted in the absence of any commercial or financial relationships that could be construed as a potential conflict of interest.

Publisher's Note: All claims expressed in this article are solely those of the authors and do not necessarily represent those of their affiliated organizations, or those of the publisher, the editors and the reviewers. Any product that may be evaluated in this article, or claim that may be made by its manufacturer, is not guaranteed or endorsed by the publisher.

Copyright (c) 2022 Beauchet, Cooper-Brown, Lubov, Allali, Afilalo and Launay. This is an open-access article distributed under the terms of the Creative Commons Attribution License (CC BY). The use, distribution or reproduction in other forums is permitted, provided the original author(s) and the copyright owner(s) are credited and that the original publication in this journal is cited, in accordance with accepted academic practice. No use, distribution or reproduction is permitted which does not comply with these terms. 